\title{
Middleterm Flares in T Tau Stars of the Tau - Aur Complex
}

\author{
Alisher Hojaev \\ Ulugbek Astronomical Institute, Tashkent 700052, Uzbekistan
}

\section{Introduction}

Light variations of different kinds appear to be one of the characteristic features of $\mathrm{T}$ Tau stars. They are mainly caused by changes in the continuum radiation. The availability of large observational databases makes it possible to investigate different patterns of variability such as stochastic, quasiperiodic and quasicyclic ones with typical time scales from days to years, as well as sporadic flare and fuorlike outbursts. Recent attempts in classification of the photometric variability of PMS-stars were made by Herbst (1994). In this paper, we study the observational results on flares in $\mathrm{T}$ Tau and related stars belonging to the Taurus-Auriga complex, which resemble that of ordinary flare stars.

\section{Observational data and results}

A census of $\mathrm{T}$ Tau stars with homogeneous observations of short and middleterm flares is presented in Table 1 . Our selection criteria were: pre- and postflare levels of brightness ought to be quasiquiescent; the amplitude has to be more than 3 times the value of standard error; the duration of brightening does not exceed a few hundred minutes; the lightcurve shape should be similar to that of a typical flare. For convenience, all observational data in Table 1 are divided into 3 subgroups.

The light curves of all flare events cannot be not distinguished from those occuring in cluster flare stars. Morphological consideration shows a presence of multiple structure (two of the FY Tau flares) as well as a simple one (another FY Tau flare, flares in V927 Tau, FZ Tau, CI Tau etc.). Estimated flare energies released in $T$ Tau stars (on the average $10^{36} \mathrm{erg}$ ) are greater than in flare stars (up to $10^{35} \mathrm{erg}$ ). The $V / B-V$ diagram for the above stated stars is shown in Fig. 1. Flaring $T$ Tau stars are apparently located somewhat lower and spread over a larger area than other objects and lie generally between $T$ Tau stars without detected flares (or with smaller frequency of flare activity) and cluster flare stars, which occupy the dotted field. 
Table 1. Flares in T Tau stars of the Tau-Aur complex

\begin{tabular}{|c|c|c|c|c|c|c|}
\hline $\begin{array}{l}\text { Star } \\
\text { name }\end{array}$ & $\begin{array}{l}\text { Flares } \\
\text { number }\end{array}$ & $\begin{array}{c}\text { JD } \\
2400000+\end{array}$ & Magnit & $\begin{array}{l}\text { Ampl. } \\
\text { [mag] }\end{array}$ & $\begin{array}{l}\text { Duration } \\
\text { [min] }\end{array}$ & Notes/Remarks \\
\hline \multirow[t]{3}{*}{ FY Tau } & 3 & 44634.29 & 16.7 & 1.5 & 60 & $\operatorname{tt}(1), \mathrm{vb}$ \\
\hline & & 45013.21 & 16.8 & 2.1 & 215 & \\
\hline & & 45286.44 & 17.1 & 1.6 & 100 & \\
\hline \multirow[t]{2}{*}{ VY Tau } & 2 & 45644.32 & 16.4 & 1.5 & 60 & $\operatorname{tt}(1), b(2)$ \\
\hline & & 45678.28 & 16.4 & 1.1 & 50 & \\
\hline \multirow[t]{2}{*}{ V955 Tau } & 2 & 44689.25 & 16.1 & 1.2 & 40 & $\operatorname{tt}(1), \mathrm{b}(2)$ \\
\hline & & 45680.45 & 16.3 & 0.7 & 15 & \\
\hline V927 Tau & 1 & 45683.49 & 16.1 & 2.1 & 55 & $\operatorname{tt}(1), b(2)$ \\
\hline FZ Tau & 1 & 45368.19 & 15.7 & 1.2 & 50 & $\operatorname{tt}(1), v b$ \\
\hline GI Tau & 1 & 45286.59 & 15.4 & 1.7 & 35 & $\operatorname{tt}(1), v b, b(2)$ \\
\hline CI Tau & 1 & 45368.26 & 15.2 & 2.3 & 90 & $\operatorname{tt}(1)$ \\
\hline HP Tau & 1 & 45013.18 & 15.5 & 0.7 & 40 & $\operatorname{tt}(1)$ \\
\hline DP Tau & 1 & 45644.38 & 16.1 & 1.0 & 30 & $\operatorname{tt}(1)$ \\
\hline FF Tau & 1 & 35041.79 & 15.8 & 1.4 & 61 & $\operatorname{wtt}(1)$ \\
\hline HV Tau & 1 & 44880.47 & 17.1 & 1.4 & 50 & $w \operatorname{tt}(1), b(2)$ \\
\hline В 13 & 1 & 44994.31 & 17.1 & 2.3 & $>50$ & $w \operatorname{tt}(1), b(2)$ \\
\hline \multirow[t]{2}{*}{ B 41} & 2 & 45289.38 & 16.1 & 1.3 & 25 & $t t ?, e-a$ \\
\hline & & 45311.23 & 16.3 & 2.1 & $>65$ & \\
\hline HQ Tau & 1 & 45644.29 & 14.6 & 1.6 & 20 & in \\
\hline GN Tau & 1 & 45724.25 & 17.3 & 1.4 & 20 & in,,$-\mathbf{a}$ \\
\hline SVS 1099 & 1 & 45373.17 & 14.5 & 1.3 & 170 & in \\
\hline V590 Tau & 1 & 44668.20 & 16.8 & 1.0 & 30 & in \\
\hline
\end{tabular}

Comments: (1) Herbig \& Bell (1988); (2) Leinert et al. (1993);

$\mathrm{b}$ - binary; vb - vis. binary; in - Orion variable; e-a - $\mathrm{H} \alpha$ in emission

Besides the indicated stars in the Tau-Aur complex, references on flares and flare-like events are available which were detected in T Tau, RY Tau, DF Tau, V410 Tau, SU Aur, DG Tau, and DP Tau, which belong to the tt- and wtt-types. T Tau is a relatively frequently flaring T Tau star $(f \approx 30 / \mathrm{h})$. Flares of DF Tau may be due to active plages on the stellar surface. Krautter et al. (1994) have shown that in Tau-Aur the ratio WTTS/CTTS $=1.3$ and close that of the Orion Nebula (1.5). These complexes are similar as concerns the flare activity of inner stars. A large percentage of flaring $T$ Tau stars exists in the Orion Complex, but only a few of these stars are found in the NGC2264 and NGC7000 aggregates.

Possible mechanisms of flare activity of $\mathrm{T}$ Tauri stars are in accordance with the evolutionary scenario outlined by Montmerle and Andre (1989): In classical T Tau stars, flares seem to be caused by outbursts on the stellar surface, 


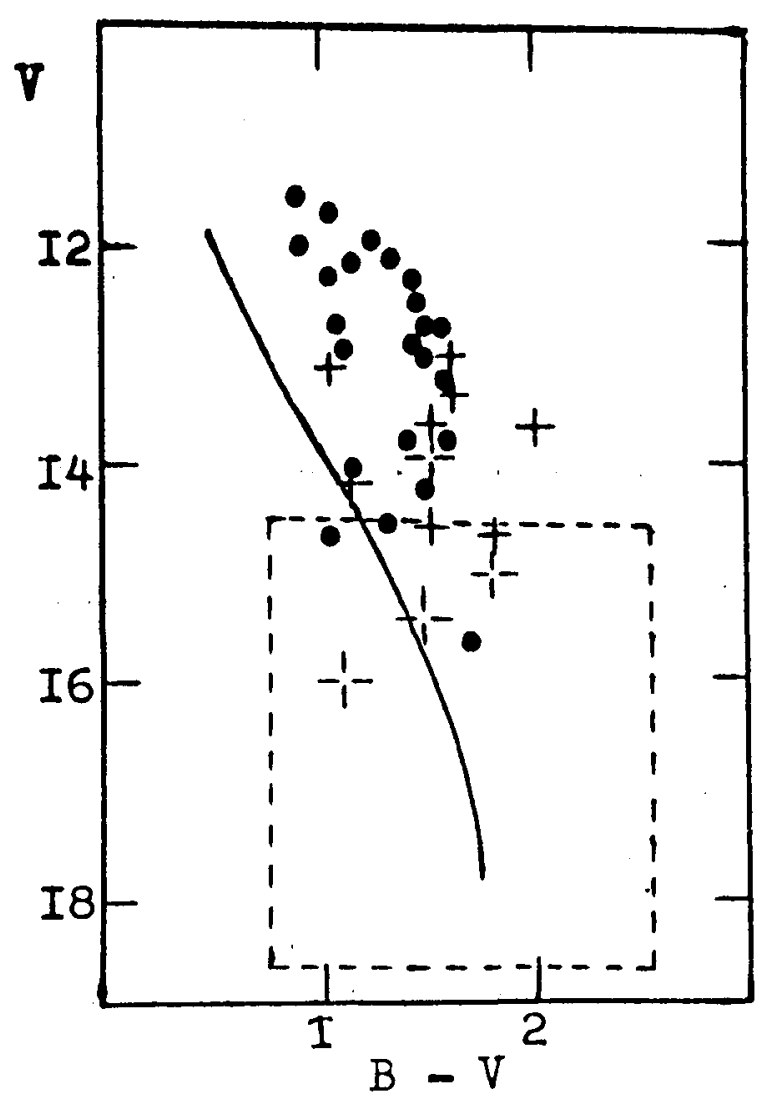

Fig. 1. The $V / B-V$ diagram for the $\mathrm{T}$ Tau stars given in Tab. 1: + marks $\mathrm{T}$ Tau stars with detected flares, - T Tau stars without flares.

but the initial radiation is distorted by circumstellar obscuration as well as by interaction processes with the surrounding accretion disk and the dust +gas envelope (sporadic outflow and infall of matter). Weak $\mathrm{T}$ Tauri stars have lost their circumstellar envelopes and their flares are presumably caused by the same mechanism which is dominant in typical flare stars.

\section{References}

Herbig G., Bell K., 1988, Lick Obs. Bull. No. 1111, p. 90.

Herbst W., 1994, in Nature and Evolutionary Status of Herbig Ae/Be stars, PASP Conference Series Vol. 62, p. 35

Krautter J., et al., 1994, Rev. Mex. Astr. Astrofis. 29, 41

Leinert Ch., et al., 1993, A\&A 278, 129

Montmerle T., Andre P., 1989, in Low Mass Star Formation and Pre-Main Sequence

Objects, ESO Conf. and Workshop Proceedings No. 33, p. 407 